\title{
Synthesis, Characterization and Antimicrobial Studies on Some Newer Imidazole Analogs
}

\author{
Rajiv DAHIYA \\ Department of Pharmaceutical Chemistry, Rajiv Academy for Pharmacy, \\ Mathura - 281001, Uttar Pradesh, India
}

\begin{abstract}
A novel series of 3,5-diiodo-4-(2-methyl-1H-imidazol-5-yl)benzoic acid analogs of amino acids, dipeptides and tripeptides was synthesized by using dicyclohexylcarbodiimide and diisopropylcarbodiimide (DCC/DIPC) as coupling agents and triethylamine (TEA) as base. Structures of all the newly synthesized compounds were confirmed by elemental analysis and IR, ${ }^{1} \mathrm{H} N M R,{ }^{13} \mathrm{C} N M R$ and mass spectral data. Imidazolopeptides were investigated for their antimicrobial activity and some of newly synthesized compounds $\mathbf{2 c}, \mathbf{2 d}, \mathbf{2 h}$ and their hydrolyzed analogs $\mathbf{3 b}$, 3d exhibited potent bioactivity against pathogenic fungi Candida albicans and dermatophytes Trichophyton mentagrophytes and Microsporum audouinii with MIC values of $12.5-6 \mu \mathrm{g} / \mathrm{ml}$, as compared to the reference drug griseofulvin. In addition, moderate activity against gram negative bacteria Pseudomonas aeruginosa and Klebsiella pneumoniae was also observed for synthesized imidazolopeptides.
\end{abstract}

\section{Keywords}

Imidazolopeptides • p-Amino-3,5-diiodobenzoic acid • Peptide coupling • Antifungal activity $•$ Antibacterial activity

\section{Introduction}

In past decades, imidazoles have proved their potential in development of pharmaceutically important organic compounds both of natural and synthetic origin 
[1]. Imidazole analogs deal with a variety of bioactivities viz. antitumor [2, 3], antiHIV [4], antimicrobial [5-8], anticonvulsant [9], antitubercular [10], antiprotozoal [11], anti-inflammatory [12], FTase and MAP kinase p38 inhibitory activities [13, 14]. Literature is enriched with lot of work on synthesis of potent substituted imidazole derivatives with diverse pharmacological activities $[15,16]$ but only few reports have been received on peptide coupling of imidazoles. Thus keeping in view the biological potency of imidazole derivatives as well as taking advantage of biodegradability and biocompatibility of amino acids/peptides and further, in continuation of our research work on synthesizing peptide derivatives of heterocyclic and other aromatic compounds [17-29], a novel series of 3,5-diiodo-4(2-methyl-1H-5-imidazolyl)benzoyl amino acids and peptides with an anticipation to get potent agents of more therapeutic efficacy with lesser adverse effects.

\section{Chemistry}

4-Amino-3,5-diiodobenzoic acid (1) was prepared according to literature procedure [30] in good yields by iodination of $p$-aminobenzoic acid (PABA) using iodine monochloride. 3,5-Diiodo-4-(2-methyl-1H-5-imidazolyl)benzoic acid (2) was synthesized by diazotization of compound 1 followed by subsequent interaction with 2-methylimidazole in presence of aqueous cupric chloride. Dipeptides Boc-his-tyr$\mathrm{OMe}$, Boc-phe-try-OMe and Boc-gly-gly-OMe were prepared from the corresponding Boc-amino acids viz. Boc-his- $\mathrm{OH}$, Boc-phe-OH and Boc-gly-OH, and amino acid methyl ester hydrochlorides such as tyr-OMe. $\mathrm{HCl}$, try-OMe. $\mathrm{HCl}$ and glyOMe. $\mathrm{HCl}$ using DCC and $\mathrm{N}$-methylmorpholine (NMM) in chloroform [31]. Similarly, tripeptides Boc-pro-val-pro-OMe, Boc-ile-phe-leu-OMe and tetrapeptide Boc-glyhis-ala-try-OMe were prepared by coupling Boc-dipeptides with corresponding amino acid methyl ester hydrochlorides and dipeptide methyl esters. All Bocdi/tri/tetrapeptide methyl esters were deprotected at amino terminals using trifluoroacetic acid (TFA) prior to coupling.

3,5-Diiodo-4-(2-methyl-1H-5-imidazolyl)benzoic acid (2) was coupled with various L-amino acid methyl ester hydrochlorides/peptide methyl esters using 
DCC/DIPC and TEA to get newly synthesized 3,5-diiodo-4-(2-methyl-1H-5imidazolyl)benzoyl amino acid methyl esters (2a-b), dipeptide methyl esters (2c-e) and tri/tetrapeptide methyl esters (2f-h). Finally, compounds $\mathbf{2} \mathbf{b}-\mathbf{c}$ and $\mathbf{2} \mathbf{g}-\mathbf{h}$ were hydrolyzed with lithium hydroxide ( $\mathrm{LiOH}$ ) to get the corresponding acid derivatives 3a-d. Structures of all the newly synthesized compounds were confirmed by IR, ${ }^{1} \mathrm{H}$ NMR, ${ }^{13} \mathrm{C}$ NMR and mass spectral data. Elemental analysis of the novel compounds was performed for carbon, hydrogen and nitrogen content. The synthesis of compounds $\mathbf{2 a - 3 d}$ is mentioned in Scheme 1 and their physicochemical data is described in Table 1.

\section{Results and Discussion}

All imidazolopeptide derivatives $\mathbf{2} \mathbf{a}-\mathbf{h}$ were synthesized in good yields using DCC/DIPC as coupling agents and TEA as base. Presence of bands at 3307-2499, 1696 and $593 \mathrm{~cm}^{-1}$ in IR spectrum of compound 2 clearly indicated presence of functional groups like $-\mathrm{COOH}$ and $-\mathrm{I}$ but no free $-\mathrm{NH}_{2}$ group which was present in starting material $p$-amino-3,5-diiodobenzoic acid (1). Furthermore, IR spectra of peptide derivatives 2a-h showed Amide I and Amide II bands at $1662-1639 \mathrm{~cm}^{-1}$ and $1538-1531 \mathrm{~cm}^{-1}$ indicating formation of peptide bonds and successfulness of coupling reaction. This fact was further confirmed by appearance of broad singlets at 9.32-6.96 ppm (for imino proton of CO-Nㅡㅡ moiety) in ${ }^{1} \mathrm{H}$ NMR spectra and singlets at $179.5-167.2 \mathrm{ppm}$ (for carbonyl carbon of $\underline{\mathrm{C}} \mathrm{O}-\mathrm{NH}$ moiety) in ${ }^{13} \mathrm{C}$ NMR spectra of compounds $\mathbf{2} \mathbf{a}-\mathbf{h}$. Mass spectra of peptide ester derivatives showed molecular ion peaks along with isotopic peaks at $m / z$ values, consistent with their respective molecular formulas. All peptide ester derivatives showed easily distinguishable $\mathrm{R}-\mathrm{C} \equiv \mathrm{O}^{+}$ion peaks at $\mathrm{M}-31$ along with characteristic fragmentation pattern after and before the carbonyl moiety in their respective structures. Furthermore, $\left[\mathrm{CH}_{3} \mathrm{O}^{+}\right]$and $\left[\mathrm{CH}_{3} \mathrm{OCO}^{+}\right]$fragment ion peaks appeared at $\mathrm{m} / \mathrm{z}$ values 31 and 59 in mass spectra of peptide ester derivatives. 
$\underset{\mathrm{OH}}{\mathrm{O}} \stackrel{\text { lodination }}{\mathrm{ICl}}$

1

Diazotization $\mathrm{NaNO}_{2}, \mathrm{HCl}$<smiles>Cc1ncc[nH]1</smiles>

$\mathrm{H}$

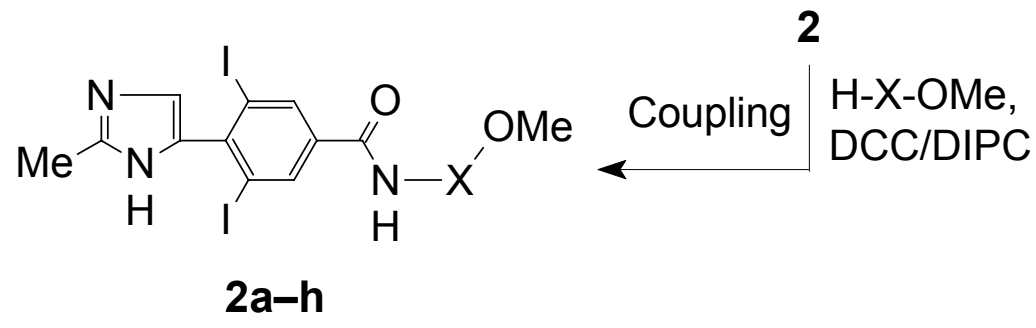

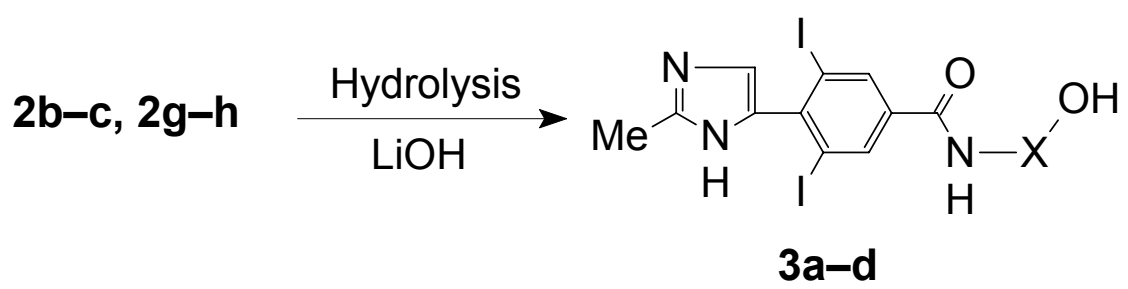

Sch. 1. $X=$ pro (2a), leu (2b), his-tyr (2c), phe-try (2d), gly-gly (2e), pro-val-pro (2f), ile-phe-leu (2g), gly-his-ala-try (2h), leu (3a), his-tyr (3b), ile-phe-leu (3c), gly-hisala-try (3d).

Structures of hydrolyzed analogs $\mathbf{3 a - d}$ were confirmed by appearance of strong bands at $1713-1708 \mathrm{~cm}^{-1}(\mathrm{C}=\mathrm{O}$ str, $\underline{\mathrm{COOH}})$ in IR spectra, broad singlets at $8.69 \mathrm{ppm}$ (for hydroxyl proton of $\mathrm{COO} \underline{\mathrm{H}}$ ) in ${ }^{1} \mathrm{H}$ NMR spectra and singlets at 175.0 ppm (for carbonyl carbon of $\underline{\mathrm{COOH}}$ ) in ${ }^{13} \mathrm{C}$ NMR spectra. This fact was further supported by disappearance of medium to strong bands at $1754-1748 \mathrm{~cm}^{-1}(\mathrm{C}=\mathrm{O}$ str, ester) and $1274-1269 \mathrm{~cm}^{-1}$ (C-O str, ester) in IR spectra and singlets at 52.5 ppm (for carbonyl carbon of $\mathrm{O}_{\mathrm{CH}} \mathrm{H}_{3}$ ) in ${ }^{13} \mathrm{C}$ NMR spectra of compounds $3 \mathbf{a}-\mathbf{d}$. 
All hydrolyzed peptide derivatives showed peaks at $\mathrm{M}-17$ and $\mathrm{M}-45$ in their mass spectra by loss of $\mathrm{OH}$ and $\mathrm{COOH}$ respectively. Moreover, $\left[\mathrm{COOH}^{+}\right]$fragment ion peak appeared at $\mathrm{m} / \mathrm{z}$ value 45 in mass spectra of compound $\mathbf{3 c}$ and $\mathbf{3 d}$ along with characteristic fragmentation pattern after and before the carbonyl moiety. Moreover, the results of elemental analysis revealed the variation by a factor of \pm 0.03 from calculated values.

\section{Biological activity}

All the newly synthesized compounds $\mathbf{2 a - 3 d}$ were evaluated for its antimicrobial activity against four bacterial strains Bacillus subtilis (NCIM 2063), Staphylococcus aureus (NCIM 2079), Pseudomonas aeruginosa (NCIM 2034) and Klebsiella pneumoniae (NCIM 2011) and four fungal strains Microsporum audouinii (MUCC 545), Trichophyton mentagrophytes (MUCC 665), Candida albicans (MUCC 29) and Aspergillus niger (MUCC 177) at 50-6 $\mathrm{g} \mathrm{ml}^{-1}$ concentration, according to modified Kirby-Bauer's disk diffusion method [32]. MIC values of test compounds were determined by tube dilution technique. The solvents DMF/DMSO were used as negative controls and ciprofloxacin/griseofulvin were used as standards. Calculated average diameters (for triplicate sets) of the zones of inhibition (in $\mathrm{mm}$ ) for test samples were compared with that produced by the standard drugs.

Almost, all the synthesized compounds were found to exhibit moderate to good antifungal activity and mild to moderate antibacterial activity. Analysis of antimicrobial data suggested that compounds $\mathbf{2 c}, \mathbf{2 d}, \mathbf{2 h}, \mathbf{3 b}$ and $\mathbf{3 d}$ possessed higher antifungal activity against dermatophytes and Candida sp. in comparison to standard drug. 
Tab. 1. Physico-chemical characterization of synthesized compounds

\begin{tabular}{|c|c|c|c|c|c|c|c|c|}
\hline \multirow[t]{2}{*}{ Compd. } & \multirow[t]{2}{*}{$\mathbf{X}$} & \multirow{2}{*}{$\begin{array}{c}\text { Mol. } \\
\text { formula } \\
\text { (Mol. wt.) }\end{array}$} & \multirow{2}{*}{$\begin{array}{l}\text { M.p. } \\
\left({ }^{\circ} \mathrm{C}\right)\end{array}$} & \multirow{2}{*}{$\begin{array}{c}\text { Yield } \\
(\%)\end{array}$} & \multirow{2}{*}{$\begin{array}{c}\mathbf{R}_{\mathbf{f}} \\
\text { Value* }^{*}\end{array}$} & \multicolumn{3}{|c|}{$\begin{array}{l}\text { Elemental analysis } \\
\text { [Calcd. (found)] }\end{array}$} \\
\hline & & & & & & $\% \mathrm{C}$ & $\% \mathrm{H}$ & $\% \mathrm{~N}$ \\
\hline 2 & - & $\begin{array}{c}\mathrm{C}_{11} \mathrm{H}_{8} \mathrm{I}_{2} \mathrm{~N}_{2} \mathrm{O}_{2} \\
(454)\end{array}$ & 118 & 72 & $0.79^{1}$ & $\begin{array}{c}29.10 \\
(29.09)\end{array}$ & $\begin{array}{c}1.78 \\
(1.80)\end{array}$ & $\begin{array}{c}6.17 \\
(6.19)\end{array}$ \\
\hline $2 a$ & pro & $\begin{array}{c}\mathrm{C}_{17} \mathrm{H}_{17} \mathrm{I}_{2} \mathrm{~N}_{3} \mathrm{O}_{3} \\
(565)\end{array}$ & - & 77 & 0.58 & \begin{tabular}{|c|}
36.13 \\
$(36.11)$ \\
\end{tabular} & $\begin{array}{c}3.03 \\
(3.06)\end{array}$ & $\begin{array}{c}7.44 \\
(7.46)\end{array}$ \\
\hline $2 b$ & leu & $\begin{array}{c}\mathrm{C}_{18} \mathrm{H}_{21} \mathrm{I}_{2} \mathrm{~N}_{3} \mathrm{O}_{3} \\
(581)\end{array}$ & 105 & 82 & 0.67 & $\begin{array}{c}37.20 \\
(37.23)\end{array}$ & $\begin{array}{c}3.64 \\
(3.62)\end{array}$ & $\begin{array}{l}7.23 \\
(7.25)\end{array}$ \\
\hline $2 c$ & his-tyr & $\begin{array}{c}\mathrm{C}_{27} \mathrm{H}_{26} \mathrm{I}_{2} \mathrm{~N}_{6} \mathrm{O}_{5} \\
(768)\end{array}$ & 120 & 75 & 0.81 & $\begin{array}{c}42.21 \\
(42.19)\end{array}$ & $\begin{array}{c}3.41 \\
(3.44)\end{array}$ & $\begin{array}{r}10.94 \\
(10.95)\end{array}$ \\
\hline $2 d$ & phe-try & $\begin{array}{c}\mathrm{C}_{32} \mathrm{H}_{29} \mathrm{I}_{2} \mathrm{~N}_{5} \mathrm{O}_{4} \\
(801)\end{array}$ & 84 & 79 & 0.74 & $\begin{array}{l}47.96 \\
(47.98)\end{array}$ & $\begin{array}{c}3.65 \\
(3.65)\end{array}$ & $\begin{array}{c}8.74 \\
(8.77)\end{array}$ \\
\hline $2 e$ & gly-gly & $\begin{array}{c}\mathrm{C}_{16} \mathrm{H}_{16} \mathrm{I}_{2} \mathrm{~N}_{4} \mathrm{O}_{4} \\
(582)\end{array}$ & 142 & 71 & 0.51 & $\begin{array}{c}33.01 \\
(33.04)\end{array}$ & $\begin{array}{l}2.77 \\
(2.80)\end{array}$ & $\begin{array}{c}9.62 \\
(9.59)\end{array}$ \\
\hline $2 f$ & pro-val-pro & $\begin{array}{c}\mathrm{C}_{27} \mathrm{H}_{33} \mathrm{I}_{2} \mathrm{~N}_{5} \mathrm{O}_{5} \\
(761)\end{array}$ & - & 80 & 0.59 & $\begin{array}{c}42.59 \\
(42.58)\end{array}$ & $\begin{array}{c}4.37 \\
(4.35)\end{array}$ & $\begin{array}{c}9.20 \\
(9.23)\end{array}$ \\
\hline $2 g$ & ile-phe-leu & $\begin{array}{c}\mathrm{C}_{33} \mathrm{H}_{41} \mathrm{I}_{2} \mathrm{~N}_{5} \mathrm{O}_{5} \\
(841)\end{array}$ & - & 84 & 0.72 & $\begin{array}{c}47.10 \\
(47.10)\end{array}$ & $\begin{array}{c}4.91 \\
(4.92)\end{array}$ & $\begin{array}{c}8.32 \\
(8.29)\end{array}$ \\
\hline $2 \mathrm{~h}$ & gly-his-ala-try & $\begin{array}{c}\mathrm{C}_{34} \mathrm{H}_{35} \mathrm{I}_{2} \mathrm{~N}_{9} \mathrm{O}_{6} \\
(919)\end{array}$ & - & 69 & $0.61^{2}$ & $\begin{array}{c}44.41 \\
(44.42)\end{array}$ & $\begin{array}{c}3.84 \\
(3.85)\end{array}$ & $\begin{array}{c}13.71 \\
(13.69)\end{array}$ \\
\hline $3 a$ & leu & $\begin{array}{c}\mathrm{C}_{17} \mathrm{H}_{19} \mathrm{I}_{2} \mathrm{~N}_{3} \mathrm{O}_{3} \\
(567)\end{array}$ & 63 & 86 & 0.49 & $\begin{array}{c}36.00 \\
(35.98)\end{array}$ & $\begin{array}{c}3.38 \\
(3.41)\end{array}$ & $\begin{array}{c}7.41 \\
(7.40)\end{array}$ \\
\hline $3 b$ & his-tyr & $\begin{array}{c}\mathrm{C}_{26} \mathrm{H}_{24} \mathrm{I}_{2} \mathrm{~N}_{6} \mathrm{O}_{5} \\
(754)\end{array}$ & 159 & 89 & 0.66 & $\begin{array}{c}41.40 \\
(41.43)\end{array}$ & $\begin{array}{c}3.21 \\
(3.19)\end{array}$ & $\begin{array}{c}11.14 \\
(11.15)\end{array}$ \\
\hline $3 c$ & ile-phe-leu & $\begin{array}{c}\mathrm{C}_{33} \mathrm{H}_{41} \mathrm{I}_{2} \mathrm{~N}_{5} \mathrm{O}_{5} \\
(841)\end{array}$ & - & 92 & 0.63 & \begin{tabular}{|c|}
46.45 \\
$(46.46)$
\end{tabular} & $\begin{array}{c}4.75 \\
(4.77)\end{array}$ & $\begin{array}{c}8.46 \\
(8.44)\end{array}$ \\
\hline $3 d$ & gly-his-ala-try & $\begin{array}{c}\mathrm{C}_{33} \mathrm{H}_{33} \mathrm{I}_{2} \mathrm{~N}_{9} \mathrm{O}_{6} \\
(905)\end{array}$ & - & 90 & $0.85^{2}$ & $\begin{array}{c}43.77 \\
(43.75)\end{array}$ & $\begin{array}{c}3.67 \\
(3.68)\end{array}$ & $\begin{array}{c}13.92 \\
(13.90)\end{array}$ \\
\hline
\end{tabular}


Tab. 2. Antimicrobial activity of synthesized compounds

\begin{tabular}{|c|c|c|c|c|c|c|c|c|}
\hline \multirow{3}{*}{ Compd. } & \multicolumn{8}{|c|}{ Diameter of zone of inhibition (mm) } \\
\hline & \multicolumn{4}{|c|}{ Bacterial strains } & \multicolumn{4}{|c|}{ Fungal strains } \\
\hline & $\begin{array}{l}\text { B. } \\
\text { sub. }\end{array}$ & $\begin{array}{l}\text { S. } \\
\text { aur. }\end{array}$ & $\begin{array}{c}P . \\
\text { aeru. }\end{array}$ & $\begin{array}{c}\text { K. } \\
\text { pneu. }\end{array}$ & $\begin{array}{l}\text { C. } \\
\text { alb. }\end{array}$ & $\begin{array}{c}\text { M. } \\
\text { audo. }\end{array}$ & $\begin{array}{c}A . \\
\text { niger }\end{array}$ & $\begin{array}{c}T . \\
\text { menta. }\end{array}$ \\
\hline $2 a$ & $9(25)^{\dagger}$ & $11(50)$ & 11(6) & $10(12.5)$ & $14(6)$ & $14(6)$ & $12(12.5)$ & $14(6)$ \\
\hline $2 b$ & 11(25) & 13(25) & $17(6)$ & 13(12.5) & $15(6)$ & $13(6)$ & $14(12.5)$ & $13(6)$ \\
\hline 2c & $10(12.5)$ & $9(50)$ & 14(6) & $11(12.5)$ & $21(6)$ & $19(6)$ & $12(12.5)$ & $21(6)$ \\
\hline 2d & $15(6)$ & $10(12.5)$ & $20(6)$ & $13(12.5)$ & $23(6)$ & $22(6)$ & $15(12.5)$ & $24(6)$ \\
\hline $2 e$ & $16(6)$ & $9(12.5)$ & $24(6)$ & 19(12.5) & $17(6)$ & $12(6)$ & $9(12.5)$ & $16(12.5)$ \\
\hline $2 f$ & $15(12.5)$ & $13(12.5)$ & $25(6)$ & $18(12.5)$ & $11(12.5)$ & $10(6)$ & $12(12.5)$ & $9(25)$ \\
\hline $2 g$ & 13(25) & $11(25)$ & $17(12.5)$ & $14(12.5)$ & $17(12.5)$ & $10(6)$ & $14(12.5)$ & $11(25)$ \\
\hline $2 h$ & 11(12.5) & $10(25)$ & $13(6)$ & 12(12.5) & $22(6)$ & $18(6)$ & $15(12.5)$ & $21(6)$ \\
\hline $3 a$ & $15(25)$ & $16(25)$ & $19(6)$ & $16(12.5)$ & $17(6)$ & $15(6)$ & $15(12.5)$ & $17(6)$ \\
\hline $3 b$ & $16(12.5)$ & $10(50)$ & $17(6)$ & $15(12.5)$ & $25(6)$ & $20(6)$ & $14(12.5)$ & $23(6)$ \\
\hline $3 c$ & $16(25)$ & $13(25)$ & $19(12.5)$ & $16(12.5)$ & $19(12.5)$ & $14(6)$ & $16(12.5)$ & $14(25)$ \\
\hline $3 d$ & $13(12.5)$ & $12(25)$ & $18(6)$ & $15(12.5)$ & $25(6)$ & $21(6)$ & $17(12.5)$ & $23(6)$ \\
\hline Control $^{\ddagger}$ & - & - & - & - & - & - & - & - \\
\hline Ciprofloxacin & $20(6)$ & $20(12.5)$ & $25(6)$ & 19(12.5) & - & - & - & - \\
\hline Griseofulvin & - & - & - & - & $20(6)$ & $17(6)$ & $18(12.5)$ & $20(6)$ \\
\hline
\end{tabular}

Moreover, all the synthesized compounds exhibited moderate activity against gram negative bacteria. Compounds $2 \mathrm{e}$ and $\mathbf{2 f}$ exhibited bioactivity equivalent or closer to reference compound, against $P$. aeruginosa and $K$. pneumoniae. Moreover, comparison of antimicrobial data further suggested that amino acid and peptide derivatives $\mathbf{3 a - d}$ possessed more antimicrobial activity as compared to corresponding methyl ester derivatives $\mathbf{2} \mathbf{b}-\mathbf{c}$ and $\mathbf{2} \mathbf{g}-\mathbf{h}$ (Table 2 ).

Newly synthesized compounds $\mathbf{2 a - 3 d}$ were found to be more potent in comparison to previously synthesized diiododimethylbenzimidazole analogs against $K$. pneumoniae and M. audouinii. Even some of the synthesized analogs $\mathbf{2 c}, \mathbf{2 d}$, 
$\mathbf{2 h}, \mathbf{3 b}$ and $\mathbf{3 d}$ displayed better activity against $P$. aeruginosa and compounds $\mathbf{2} \mathbf{d}-\mathbf{f}$ exhibited better activity against $C$. albicans when compared to their corresponding dimethylbenzimidazolopeptides [18]. However, activity of compounds 2a-3d against gram positive bacteria was found to be less in comparison to earlier synthesized analogs. Moreover, presence of iodine atoms in nucleus was found to confer more antimicrobial activity especially against $P$. aeruginosa, $K$. pneumoniae, C. albicans and dermatophytes, as evident from previously synthesized noniodinated benzimidazolopeptide analogs [26].

\section{Experimental}

Melting points were determined by open capillary method and are uncorrected. L-amino acids, DIPC, DCC, TFA, NMM and TEA were procured from SpectroChem Ltd, Mumbai, India. IR spectra were recorded on Shimadzu 8700 fourier transform infrared spectrophotometer using a thin film supported on $\mathrm{KBr}$ pellets for solids and $\mathrm{CHCl}_{3}$ as solvent for semisolids. ${ }^{1} \mathrm{H}$ NMR and ${ }^{13} \mathrm{C}$ NMR spectra were recorded on Bruker AC NMR spectrometer (300 and $50 \mathrm{MHz}$ ) using DMSO- $d_{6}$ and $\mathrm{CDCl}_{3}$ as solvents and TMS as internal standard. Mass spectra were recorded on Jeol JMS DX 303 Mass spectrometer operating at $70 \mathrm{eV}$. Elemental analysis of all compounds was performed on Elementar vario EL III. Purity of all the compounds was checked by TLC on precoated silica gel G plates.

\section{Preparation of peptides}

Amino acid methyl ester hydrochloride/dipeptide methyl ester $(0.01 \mathrm{~mol})$ was dissolved in $\mathrm{CHCl}_{3}(15 \mathrm{ml})$. To this, $\mathrm{NMM}(0.021 \mathrm{~mol})$ was added at $0{ }^{\circ} \mathrm{C}$ and the reaction mixture was stirred for $10 \mathrm{~min}$. Boc-amino acid/dipeptide $(0.01 \mathrm{~mol})$ in $\mathrm{CHCl}_{3}(15 \mathrm{ml})$ and $\mathrm{DCC}(0.01 \mathrm{~mol})$ were added with stirring. After $36 \mathrm{~h}$, the reaction mixture was filtered and the residue was washed with $\mathrm{CHCl}_{3}(30 \mathrm{ml})$ and added to the filtrate. The filtrate was washed with $25 \mathrm{ml}$ each of $5 \% \mathrm{NaHCO}_{3}$ and saturated $\mathrm{NaCl}$ solutions. The organic layer was dried over anhydrous $\mathrm{Na}_{2} \mathrm{SO}_{4}$, filtered and evaporated in vacuum. The crude product was crystallized from a mixture of 
chloroform and $n$-hexane followed by cooling at $0^{\circ} \mathrm{C}$. Resulting Bocdi/tri/tetrapeptide methyl ester $(0.01 \mathrm{~mol})$ was dissolved in $\mathrm{CHCl}_{3}(15 \mathrm{ml})$ and treated with trifluoroacetic acid (TFA, $0.02 \mathrm{~mol}$ ). The mixture was stirred at RT for $1 \mathrm{~h}$ and washed with $25 \mathrm{ml}$ of saturated $\mathrm{NaHCO}_{3}$ solution. The resulting organic layer was dried over anhydrous $\mathrm{Na}_{2} \mathrm{SO}_{4}$ and concentrated under reduced pressure. Finally, crude product was purified by recrystallization with $\mathrm{CHCl}_{3}$ and petroleum ether (b.p. $40-60^{\circ} \mathrm{C}$ ) to get the deprotected di/tri/tetrapeptide methyl esters.

\section{Preparation of 3,5-diiodo-4-(2-methyl-1H-imidazol-5-yl)benzoic acid (2)}

A mixture of 4-amino-3,5-diiodobenzoic acid (1) (38.9 g, $0.1 \mathrm{~mol})$, dil. hydrochloric acid $(15 \%, 60 \mathrm{ml})$ and water $(90 \mathrm{ml})$ was heated to get a clear solution. After cooling, the solution was diazotized by addition of sodium nitrite solution $(30 \%, 24 \mathrm{ml})$. To the filtrate of diazotized salt solution, 2-methylimidazole $(8.2 \mathrm{~g}, 0.1$ $\mathrm{mol}$ ) and aqueous cupric chloride solution $(2.5 \mathrm{~g}$ in $10 \mathrm{ml}$ of water) were added with stirring followed by slow addition of water $(50 \mathrm{ml})$. Stirring was continued for $4 \mathrm{~h}$ and kept overnight in the refrigerator. The separated solid was collected by filtration and washed with cold water. The crude product was recrystallized from acetone to get the title compound. White crystals; m.p. $118{ }^{\circ} \mathrm{C}$; IR $(\mathrm{KBr}) v\left(\mathrm{~cm}^{-1}\right): 3488(\mathrm{~N}-\mathrm{H}$ str, ring), 3307-2499 (O-H str, COOH), 3074, 3054 (Ar-H str), 2969, 2873 (C-H str, $\mathrm{CH}_{3}$ ), 1696 (C=O str, $\left.\mathrm{COOH}\right)$, 1587, 1425, 1422 (skeletal bands), 869, 833, 748 (C-H def, out-of-plane (oop)), 593 (C-I str); ${ }^{1} \mathrm{H}-\mathrm{NMR}$ (DMSO-d $\left.\mathrm{d}_{6}\right) \delta$ (ppm): $9.22(2 \mathrm{H}$, br. s, $\mathrm{N} \underline{\mathrm{H}}$, imz and $\mathrm{O} \underline{\mathrm{H}}, \mathrm{COO} \underline{\mathrm{H}}), 8.15(2 \mathrm{H}, \mathrm{s}, \mathrm{H}-2$ and $\mathrm{H}-6$, iodobenzoic acid moiety (iba)), $7.45\left(1 \mathrm{H}, \mathrm{s}, \mathrm{H}-\delta\right.$, imidazole moiety (imz)), $2.64\left(3 \mathrm{H}, \mathrm{s}, \beta-\underline{\mathrm{H}}_{3}, \mathrm{imz}\right) ;{ }^{13} \mathrm{C}-\mathrm{NMR}$ $\left(\mathrm{CDCl}_{3}\right) \delta(\mathrm{ppm}): 170.2$ (C=O, $\left.\mathrm{COOH}\right), 159.0$ (C-2, imz), 150.1 (C-4, imz), 149.3 (C4, iba), 146.7 (C-5, imz), 141.2 (2C, C-2 and C-6, iba), 124.4 (C-1, iba), 105.8 (2C, C-3 and C-5, iba), $18.9\left(\beta-\underline{C} \mathrm{H}_{3}, \mathrm{imz}\right)$.

General procedure for synthesis of 3,5-diiodo-4-(2-methyl-1H-imidazol5-yl)benzoyl amino acid and peptide methyl esters (2a-h) 
To a mixture of amino acid methyl ester hydrochloride/di/tri/tetrapeptide methyl ester $(0.01 \mathrm{~mol})$ in $50 \mathrm{ml}$ of DMF, $2.8 \mathrm{ml}$ of TEA was added at $0{ }^{\circ} \mathrm{C}$ with stirring. Compound $2(4.54 \mathrm{~g}, 0.01 \mathrm{~mol})$ in DMF $(50 \mathrm{ml})$ and DCC/DIPC $(0.01 \mathrm{~mol})$ were added to the above mixture and stirring was done for $24 \mathrm{~h}$. After this, reaction mixture was filtered and filtrate was diluted with equal proportion of water and aqueous layer was washed with ether $(3 \times 50 \mathrm{ml})$. The organic layer was separated and dried over anhydrous $\mathrm{Na}_{2} \mathrm{SO}_{4}$, filtered and evaporated. The product obtained was dissolved in chloroform, washed with $30 \mathrm{ml}$ each of $10 \% \mathrm{HCl}$, saturated $\mathrm{NaHCO}_{3}$ solution and water followed by evaporation in vacuum. Crude product was crystallized from a mixture of ethyl acetate and petroleum ether (b.p. $40-60^{\circ} \mathrm{C}$ ).

\section{Methyl 1-[3,5-diiodo-4-(2-methyl-1H-imidazol-5-yl)benzoyl]-L-prolinate (2a)}

IR $\left(\mathrm{CHCl}_{3}\right) \vee\left(\mathrm{cm}^{-1}\right): 3486(\mathrm{~N}-\mathrm{H}$ str, ring), 3077, 3052 (Ar-H str), 2997-2992 (C-H str, $\mathrm{CH}_{2}$, pro), 2968, 2875 ( $\mathrm{C}-\mathrm{H}$ str, $\mathrm{CH}_{3}$ ), 1744 ( $\mathrm{C}=\mathrm{O}$ str, ester), 1658 ( $\mathrm{C}=\mathrm{O}$ str, $3^{\circ}$ amide), 1589, 1421 (skeletal bands), 1272 (C-O str, ester), 865, 838, 752 (C-H def, out-of-plane (oop)), 590 (C-I str); ${ }^{1} \mathrm{H}-N M R$ (DMSO-d $\left.d_{6}\right) \delta(\mathrm{ppm}): 8.32(1 \mathrm{H}$, s, $\mathrm{H}-\delta, i m z), 8.20(2 \mathrm{H}, \mathrm{s}, \mathrm{H}-2$ and $\mathrm{H}-6, \mathrm{iba}), 7.59(1 \mathrm{H}, \mathrm{br} . \mathrm{s}, \mathrm{N} \underline{\mathrm{H}}, \mathrm{imz}), 4.17(1 \mathrm{H}, \mathrm{t}, \mathrm{J}$ $=6.9 \mathrm{~Hz}, \mathrm{H}-\alpha$, pro), $3.72\left(2 \mathrm{H}, \mathrm{t}, J=7.15 \mathrm{~Hz}, \mathrm{H}-\delta\right.$, pro), $3.62\left(3 \mathrm{H}, \mathrm{s}, \mathrm{OC}_{3}\right), 2.65$ $\left(3 \mathrm{H}, \mathrm{s}, \beta-\underline{\mathrm{H}}_{3}, \mathrm{imz}\right), 2.20-2.14\left(2 \mathrm{H}, \mathrm{m}, \mathrm{H}-\gamma\right.$, pro), 2.05-2.01 (2H, q, H- $\beta$, pro); ${ }^{13} \mathrm{C}-$ $\operatorname{NMR}\left(\mathrm{CDCl}_{3}\right) \delta(\mathrm{ppm}): 174.4$ ( $\mathrm{C}=\mathrm{O}$, pro), 173.9 (C=O, ArçO), 159.0 (C-2, imz), 151.7 (C-4, iba), 146.6 (C-5, imz), 141.2 (2C, C-2 and C-6, iba), 137.9 (C-1, iba), 137.4 (C-4, imz), 102.6 (2C, C-3 and C-5, iba), 60.2 (C- $\alpha$, pro), $54.4\left(\mathrm{O}_{\mathrm{C}} \mathrm{H}_{3}\right), 50.1$ (C- $\delta$, pro), 28.9 (C- $\beta$, pro), 27.0 (C- $\gamma$, pro), $19.1\left(\beta-{ }^{-} \mathrm{H}_{3}, \mathrm{imz}\right)$.

\section{Methyl 1-[3,5-diiodo-4-(2-methyl-1H-imidazol-5-yl)benzoyl]-L-leucinate (2b)}

IR $(\mathrm{KBr}) \vee\left(\mathrm{cm}^{-1}\right): 3489(\mathrm{~N}-\mathrm{H}$ str, ring), 3134 ( $\mathrm{NH}$ str, amide), 3075, 3048 (Ar-H str), 2966 (C-H str, $\mathrm{CH}_{3}$ ), 2929, 2853 (C-H str, $\mathrm{CH}_{2}$ ), 1742 (C=O str, ester), 1641 ( $\mathrm{C}=\mathrm{O}$ str, $2^{\circ}$ amide), 1586, 1424 (skeletal bands), 1533 ( $\mathrm{N}-\mathrm{H}$ def, amide), 1385, 1362 (C-H bend, propyl-i), 1269 (C-O str, ester), 862, 839, 756 (C-H def, oop), 588 (C-I str); ${ }^{1} \mathrm{H}-\mathrm{NMR}$ (DMSO- $\left.d_{6}\right) \delta$ (ppm): 8.35 (2H, s, H-2 and H-6, iba), 


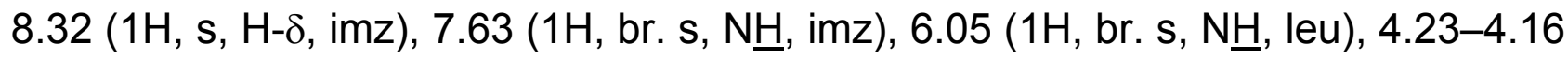
$\left(1 \mathrm{H}, \mathrm{m}, \mathrm{H}-\alpha\right.$, leu), $3.60\left(3 \mathrm{H}, \mathrm{s}, \mathrm{OC}_{3}\right), 2.68\left(3 \mathrm{H}, \mathrm{s}, \beta-\underline{\mathrm{H}}_{3}, \mathrm{imz}\right), 1.50-1.44(3 \mathrm{H}, \mathrm{m}$, $\mathrm{H}-\beta$ and $\mathrm{H}-\gamma$, leu $), 0.95(6 \mathrm{H}, \mathrm{d}, \mathrm{J}=6.2 \mathrm{~Hz}, \mathrm{H}-\delta$, leu $) ;{ }^{13} \mathrm{C}-\mathrm{NMR}\left(\mathrm{CDCl}_{3}\right) \delta(\mathrm{ppm})$ : 179.5 (C=O, leu), 168.9 (C=O, ArcO), 159.2 (C-2, imz), 152.1 (C-4, iba), 145.9 (C5, imz), 140.4 (2C, C-2 and C-6, iba), 138.2 (C-1, iba), 136.7 (C-4, imz), 104.8 (2C, C-3 and C-5, iba), $52.5\left(\mathrm{O}_{\mathrm{C}} \mathrm{H}_{3}\right), 51.3$ (C- $\alpha$, leu ), 45.5 (C- $\beta$, leu), 27.2 (C- $\gamma$, leu ), 26.4 (2C, C- $\delta$, leu), $19.4\left(\beta-\underline{C} \mathrm{H}_{3}, \mathrm{imz}\right)$.

\section{Methyl 1-[3,5-diiodo-4-(2-methyl-1H-imidazol-5-yl)benzoyl]-L-histidyl-L- tyrosinate (2c)}

$\mathrm{IR}(\mathrm{KBr}) \vee\left(\mathrm{cm}^{-1}\right)$ : 3488, $3483(\mathrm{~N}-\mathrm{H}$ str, rings), $3359(\mathrm{O}-\mathrm{H}$ str, $\mathrm{Ar}-\mathrm{OH}), 3079-$ 3075, 3055-3051 (Ar-H str), 2967, 2872 (C-H str, $\mathrm{CH}_{3}$ ), 2932, 2928, 2855, 2851 (C-H str, $\mathrm{CH}_{2}$ ), 1749 ( $\mathrm{C}=\mathrm{O}$ str, ester), 1645-1639 ( $\mathrm{C}=\mathrm{O}$ str, amide), 1592-1588, 1426-1419 (skeletal bands), 1270 (C-O str, ester), 1224 (C-O str, phenolic), 867863, 835, 788, 750, 654 (C-H def, oop), 589 (C-I str); ${ }^{1} \mathrm{H}-\mathrm{NMR}\left(\mathrm{CDCl}_{3}\right) \delta$ (ppm):

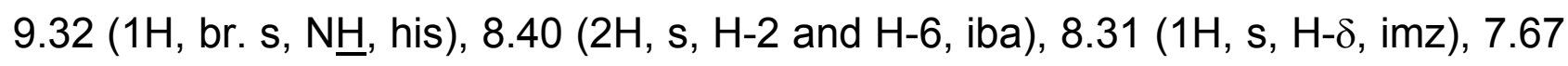
$(1 \mathrm{H}, \mathrm{s}, \mathrm{H}-4$, his), $7.55(3 \mathrm{H}, \mathrm{br} . \mathrm{s}, \underline{\mathrm{H}}$, imz, his and $\mathrm{O} \underline{\mathrm{H}}, \mathrm{tyr}), 7.38(1 \mathrm{H}, \mathrm{d}, \mathrm{J}=7.75 \mathrm{~Hz}$, $\mathrm{H}-2$, his), $6.96(1 \mathrm{H}$, br. s, N $\underline{\mathrm{H}}, \mathrm{tyr}), 6.89(2 \mathrm{H}, \mathrm{dd}, \mathrm{J}=8.6,5.25 \mathrm{~Hz}, \mathrm{H}-\mathrm{o}, \mathrm{tyr}), 6.77$ $(2 \mathrm{H}, \mathrm{dd}, \mathrm{J}=8.65,4.9 \mathrm{~Hz}, \mathrm{H}-\mathrm{m}, \mathrm{tyr}), 5.05-5.01(1 \mathrm{H}, \mathrm{m}, \mathrm{H}-\alpha$, his $), 3.94-3.89(1 \mathrm{H}, \mathrm{m}$, $\mathrm{H}-\alpha, \operatorname{tyr}), 3.56\left(3 \mathrm{H}, \mathrm{s}, \mathrm{OC}_{-} \underline{3}_{3}\right), 3.02(2 \mathrm{H}, \mathrm{d}, \mathrm{J}=5.45 \mathrm{~Hz}, \mathrm{H}-\beta, \mathrm{tyr}), 2.94(2 \mathrm{H}, \mathrm{d}, \mathrm{J}=5.8$ $\mathrm{Hz}, \mathrm{H}-\beta$, try), $2.66\left(3 \mathrm{H}, \mathrm{s}, \beta-\underline{\mathrm{H}}_{3}, \mathrm{imz}\right) ;{ }^{13} \mathrm{C}-\mathrm{NMR}\left(\mathrm{CDCl}_{3}\right) \delta$ (ppm): 172.3 (C=O, his), 171.6 (C=O, ArcO), 170.1 (C=O, tyr), 159.0 (C-2, imz), 153.9 (C-p, tyr), 150.4 (C-4, iba), 148.8 (C-2, his), 145.0 (C-5, imz), 143.6 (2C, C-m, tyr), 139.2 (2C, C-2 and C6, iba), 137.1 (C-1, iba), 136.5 (C-4, imz), 135.4 (C-4, his), 129.7 (2C, C-o, tyr), 126.5 (C- $\gamma$, tyr), 117.2 (C-5, his), 105.5 (2C, C-3 and C-5, iba), 60.3 (C- $\alpha$, his), 56.1 (C- $\alpha$, tyr), $52.8\left(\mathrm{O}_{\mathrm{C}} \mathrm{H}_{3}\right), 38.5$ (C- $\beta$, tyr), 20.2 (C- $\beta$, his), 19.1 ( $\left.\beta-\underline{\mathrm{CH}}_{3}, \mathrm{imz}\right)$.

Methyl 1-[3,5-diiodo-4-(2-methyl-1H-imidazol-5-yl)benzoyl]-L-phenylalanyl-Ltryptophanate $(2 d)$ 
IR (KBr) $v\left(\mathrm{~cm}^{-1}\right): 3487,3483(\mathrm{~N}-\mathrm{H}$ str, rings), 3138-3135 ( $\mathrm{NH}$ str, amide), 3079-3075, 3049-3046 (Ar-H str), 2965 (C-H str, $\mathrm{CH}_{3}$ ), 2927, 2923, 2855, 2851 (C-H str, $\mathrm{CH}_{2}$ ), 1746 (C=O str, ester), 1644-1639 (C=O str, amide), 1589-1585, 1428-1422 (skeletal bands), 1538, 1532 ( $\mathrm{N}-\mathrm{H}$ def, amide), 1267 (C-O str, ester), 868-861, 839, 759-756, 685 (C-H def, oop), 585 (C-I str); ${ }^{1} \mathrm{H}-\mathrm{NMR}$ (DMSO-d ${ }_{6}$ ) $\delta$ (ppm): $9.35(1 \mathrm{H}$, br. s, $\mathrm{N} \underline{\mathrm{H}}, \mathrm{phe}), 8.42(2 \mathrm{H}, \mathrm{s}, \mathrm{H}-2$ and $\mathrm{H}-6$, iba), $8.30(1 \mathrm{H}, \mathrm{s}, \mathrm{H}-\delta$, imz), $8.27(2 \mathrm{H}$, br. s, $\mathrm{N} \underline{\mathrm{H}}, \mathrm{imz}$ and try $), 7.50(1 \mathrm{H}, \mathrm{d}, \mathrm{J}=7.8 \mathrm{~Hz}, \mathrm{H}-2, \operatorname{try}), 7.22(2 \mathrm{H}$, $\mathrm{tt}, \mathrm{J}=7.8,4.65 \mathrm{~Hz}, \mathrm{H}-\mathrm{m}$, phe), 7.16-7.04 $(4 \mathrm{H}, \mathrm{m}, \mathrm{H}-4-7$, try), $6.99(1 \mathrm{H}, \mathrm{t}, \mathrm{J}=6.15$

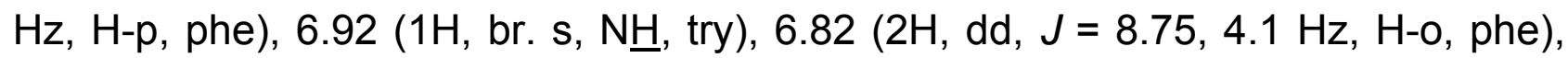
4.60-4.56 (1H, m, H- $\alpha$, phe), 4.22-4.17 (1H, m, H- $\alpha$, try), $3.56\left(3 \mathrm{H}, \mathrm{s}, \mathrm{OC}_{3}\right), 3.20$ $(2 \mathrm{H}, \mathrm{d}, \mathrm{J}=5.75 \mathrm{~Hz}, \mathrm{H}-\beta, \operatorname{try}), 2.89(2 \mathrm{H}, \mathrm{d}, \mathrm{J}=5.6 \mathrm{~Hz}, \mathrm{H}-\beta$, phe $), 2.66\left(3 \mathrm{H}, \mathrm{s}, \beta-\mathrm{C}_{3}\right.$, imz); ${ }^{13} \mathrm{C}-\mathrm{NMR}\left(\mathrm{CDCl}_{3}\right) \delta$ (ppm): 175.2 (C=O, try), 172.6 (C=O, phe), 165.4 (C=O, ArçO), 158.7 (C-2, imz), 152.0 (C-4, iba), 140.8 (C- $\gamma$, phe), 144.4 (C-5, imz), 140.1 (2C, C-2 and C-6, iba), 138.3 (C-1, iba), 137.8 (C-4, imz), 136.2 (C-2', try), 130.4 (2C, C-o, phe), 129.2 (C-2', try), 128.6 (2C, C-m, phe), 127.1 (C-p, phe), 122.9 (C2, try), 121.6, 119.5, 118.2 (3C, C-6, C-5, C-4, try), 112.4, 109.5 (2C, C-7 and C-3,

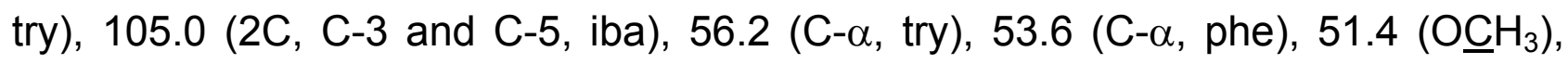
40.8 (C- $\beta$, phe), 28.3 (C- $\beta$, try), 19.1 ( $\left.\beta-\underline{C} \mathrm{H}_{3}, \mathrm{imz}\right)$.

Methyl N-[3,5-diiodo-4-(2-methyl-1H-imidazol-5-yl)benzoyl]glycylglycinate (2e)

IR (KBr) $v\left(\mathrm{~cm}^{-1}\right): 3488(\mathrm{~N}-\mathrm{H}$ str, ring), 3075, 3050 (Ar-H str), 2969, 2870 (C-H str, $\left.\mathrm{CH}_{3}\right), 2928,2923,2856,2852\left(\mathrm{C}-\mathrm{H}\right.$ str, $\left.\mathrm{CH}_{2}\right), 1747$ (C=O str, ester), 1644-1640 (C=O str, amide), 1587, 1425 (skeletal bands), 1536, 1532 ( $\mathrm{N}-\mathrm{H}$ def, amide), 1268 (C-O str, ester), 868, 839, 751 (C-H def, oop), 592 (C-I str); ${ }^{1} \mathrm{H}-\mathrm{NMR}$ (DMSO-d $\left.d_{6}\right) \delta$ (ppm): $8.65(1 \mathrm{H}$, br. s, N트, gly-2), $8.46(1 \mathrm{H}$, br. s, Nㅡ, gly-1), 8.42 (2H, s, H-2 and H-6, iba), 8.30 (1H, s, H- $\delta, i m z), 7.65$ (1H, br. s, N트, imz), $4.05(2 \mathrm{H}$, $\mathrm{d}, \mathrm{J}=5.15 \mathrm{~Hz}, \mathrm{H}-\alpha$, gly-2), $3.90\left(2 \mathrm{H}, \mathrm{d}, \mathrm{J}=5.2 \mathrm{~Hz}, \mathrm{H}-\alpha\right.$, gly-1), $3.53\left(3 \mathrm{H}, \mathrm{s}, \mathrm{OC}_{3} \underline{\mathrm{H}_{3}}\right)$, $2.69\left(3 \mathrm{H}, \mathrm{s}, \beta-\mathrm{C}_{3}, \mathrm{imz}\right) ;{ }^{13} \mathrm{C}-\mathrm{NMR}\left(\mathrm{CDCl}_{3}\right) \delta(\mathrm{ppm}): 176.6$ (C=O, gly-1), 170.2 (C=O, gly-2), 168.4 (C=O, ArㅁO), 158.7 (C-2, imz), 151.6 (C-4, iba), 146.3 (C-5, 
imz), 141.2 (2C, C-2 and C-6, iba), 138.8 (C-1, iba), 137.4 (C-4, imz), 105.0 (2C, C3 and $\mathrm{C}-5$, iba), $54.5\left(\mathrm{O} \underline{C H}_{3}\right), 46.3$ (C- $\alpha$, gly-1), 41.4 (C- $\alpha$, gly-2), $17.8\left(\beta-\underline{C} \mathrm{H}_{3}, \mathrm{imz}\right)$.

\section{Methyl 1-[3,5-diiodo-4-(2-methyl-1H-imidazol-5-yl)benzoyl]-L-prolyl-L-valyl-L- prolinate (2f)}

IR $\left(\mathrm{CHCl}_{3}\right) v\left(\mathrm{~cm}^{-1}\right): 3486(\mathrm{~N}-\mathrm{H}$ str, ring), $3136(\mathrm{~N}-\mathrm{H}$ str, amide), 3078, 3047 (Ar-H str), 2998-2993 (C-H str, $\mathrm{CH}_{2}$, pro), 2969, $2872\left(\mathrm{C}-\mathrm{H}\right.$ str, $\left.\mathrm{CH}_{3}\right), 1746(\mathrm{C}=\mathrm{O}$ str, ester), 1662-1657, 1643 ( $\mathrm{C}=\mathrm{O}$ str, $3^{\circ}$ and $2^{\circ}$ amide), 1588, 1426 (skeletal bands), 1535 ( $\mathrm{N}-\mathrm{H}$ def, amide), 1386, 1360 (C-H bend, propyl-i), 1269 (C-O str, ester), 863, 839, 755 (C-H def, oop), 587 (C-I str); ${ }^{1} \mathrm{H}-\mathrm{NMR}$ (DMSO-d $\left.\mathrm{d}_{6}\right) \delta$ (ppm): $9.25(1 \mathrm{H}$, br. s, N트, val), $8.36(2 \mathrm{H}, \mathrm{s}, \mathrm{H}-2$ and $\mathrm{H}-6$, iba), $8.30(1 \mathrm{H}, \mathrm{s}, \mathrm{H}-\delta, \mathrm{imz}), 7.63$ $(1 \mathrm{H}$, br. s, N트, imz), $4.70(1 \mathrm{H}, \mathrm{dd}, \mathrm{J}=5.9,4.35 \mathrm{~Hz}, \mathrm{H}-\alpha$, val), $4.08(1 \mathrm{H}, \mathrm{t}, \mathrm{J}=6.9$ $\mathrm{Hz}, \mathrm{H}-\alpha$, pro-1), $3.66(1 \mathrm{H}, \mathrm{t}, \mathrm{J}=6.85 \mathrm{~Hz}, \mathrm{H}-\alpha, \operatorname{pro}-2), 3.61\left(3 \mathrm{H}, \mathrm{s}, \mathrm{OC}_{3}\right), 3.45(2 \mathrm{H}$, $\mathrm{t}, J=7.15 \mathrm{~Hz}, \mathrm{H}-\delta$, pro-1), $3.45\left(2 \mathrm{H}, \mathrm{t}, J=7.2 \mathrm{~Hz}, \mathrm{H}-\delta\right.$, pro-2), $2.65\left(3 \mathrm{H}, \mathrm{s}, \beta_{-} \underline{\mathrm{C}}_{3}\right.$, imz), 2.62 (2H, q, H- $\beta$, pro-1), 2.10-2.05 (1H, m, H- $\beta$, val), 2.02-1.96 $(4 \mathrm{H}, \mathrm{m}, \mathrm{H}-\gamma$, pro-1 and pro-2), $1.93\left(2 \mathrm{H}, \mathrm{q}, \mathrm{H}-\beta\right.$, pro-2), $1.05\left(6 \mathrm{H}, \mathrm{d}, \mathrm{J}=4.65 \mathrm{~Hz}, \mathrm{H}-\gamma\right.$, val); ${ }^{13} \mathrm{C}-$ $\operatorname{NMR}\left(\mathrm{CDCl}_{3}\right) \delta(\mathrm{ppm}): 176.4(\mathrm{C}=\mathrm{O}$, pro-1), 173.1 ( $\mathrm{C}=\mathrm{O}$, pro-2), $170.8(\mathrm{C}=\mathrm{O}, \mathrm{Ar} \underline{\mathrm{CO}})$, 167.2 (C=O, val), 159.0 (C-2, imz), 150.9 (C-4, iba), 146.6 (C-5, imz), 141.5 (2C, C2 and C-6, iba), 138.0 (C-1, iba), 136.6 (C-4, imz), 104.2 (2C, C-3 and C-5, iba), 66.2 (C- $\alpha$, pro-1), 60.7 (C- $\alpha$, pro-2), $54.2\left(\mathrm{OCH}_{3}\right), 53.0$ (C- $\alpha$, phe), 45.3 (C- $\delta$, pro-2), 43.1 (C- $\delta$, pro-1), 30.2 (C- $\beta$, val), 29.3 (C- $\beta$, pro-2), 27.7 (C- $\beta$, pro-1), 27.0 (C- $\gamma$, pro-1), 23.5 (C-y, pro-2), 19.8 (2C, C-ү, val), $18.8\left(\beta-\underline{C}_{3}, \mathrm{imz}\right)$.

\section{Methyl 1-[3,5-diiodo-4-(2-methyl-1H-imidazol-5-yl)benzoyl]-L-isoleucyl-L- phenylalanyl-L-leucinate (2g)}

IR $\left(\mathrm{CHCl}_{3}\right) \vee\left(\mathrm{cm}^{-1}\right): 3484$ ( $\mathrm{N}-\mathrm{H}$ str, ring), 3079, 3052-3047 (Ar-H str), 2973, 2968, 2870 (C-H str, $\mathrm{CH}_{3}$ ), 2929-2925, 2855-2852 (C-H str, $\left.\mathrm{CH}_{2}\right), 1748$ (C=O str, ester), 1647-1642 (C=O str, amide), 1587, 1422 (skeletal bands), 1538-1531 ( $\mathrm{N}-\mathrm{H}$ def, amide), 1386, 1360 (C-H bend, propyl-i), 1274 (C-O str, ester), 869, 832, 786781, 748 (C-H def, oop), 590 (C-I str); ${ }^{1} \mathrm{H}-\mathrm{NMR}$ (DMSO-d $\left.{ }_{6}\right) \delta$ (ppm): 8.39 (2H, s, H- 
2 and $\mathrm{H}-6$, iba), $8.30(1 \mathrm{H}, \mathrm{s}, \mathrm{H}-\delta$, imz), $7.98(1 \mathrm{H}$, br. s, N트, phe), $7.65(1 \mathrm{H}$, br. s, $\mathrm{N} \underline{\mathrm{H}}, \mathrm{imz}), 7.20(2 \mathrm{H}, \mathrm{tt}, \mathrm{J}=7.75,4.8 \mathrm{~Hz}, \mathrm{H}-\mathrm{m}, \mathrm{phe}), 7.12$ (1H, br. s, N트, phe), 6.98 $(1 \mathrm{H}, \mathrm{t}, \mathrm{J}=6.2 \mathrm{~Hz}, \mathrm{H}-\mathrm{p}$, phe), $6.84(2 \mathrm{H}, \mathrm{dd}, \mathrm{J}=8.7,4.15 \mathrm{~Hz}, \mathrm{H}-\mathrm{o}, \mathrm{phe}), 6.74(1 \mathrm{H}, \mathrm{br}$. s, N트, leu), $4.59(1 \mathrm{H}, \mathrm{dd}, J=5.35,3.7 \mathrm{~Hz}, \mathrm{H}-\alpha$, ile), $4.32(1 \mathrm{H}, \mathrm{q}, \mathrm{H}-\alpha$, phe), 3.60 $\left(3 \mathrm{H}, \mathrm{s}, \mathrm{OC}_{3}\right), 3.48(1 \mathrm{H}, \mathrm{q}, \mathrm{H}-\alpha, \mathrm{leu}), 2.80(2 \mathrm{H}, \mathrm{d}, \mathrm{J}=5.65 \mathrm{~Hz}, \mathrm{H}-\beta$, phe $), 2.62(3 \mathrm{H}$, $\left.\mathrm{s}, \beta-\underline{H}_{3}, \mathrm{imz}\right), 2.02-1.96(1 \mathrm{H}, \mathrm{m}, \mathrm{H}-\beta$, ile), 1.65-1.59 (2H, $\mathrm{m}, \mathrm{H}-\mathrm{\gamma}$, ile), 1.50-1.43 $\left(3 \mathrm{H}, \mathrm{m}, \mathrm{H}-\beta\right.$ and $\mathrm{H}-\mathrm{\gamma}$, leu), $1.02\left(3 \mathrm{H}, \mathrm{d}, \mathrm{J}=5.9 \mathrm{~Hz}, \mathrm{H}-\mathrm{\gamma}^{\prime}\right.$, ile), $0.98(3 \mathrm{H}, \mathrm{d}, \mathrm{J}=5.9 \mathrm{~Hz}$, $\mathrm{H}-\delta$, ile), $0.95\left(6 \mathrm{H}, \mathrm{d}, \mathrm{J}=6.25 \mathrm{~Hz}, \mathrm{H}-\delta\right.$, leu); ${ }^{13} \mathrm{C}-\mathrm{NMR}\left(\mathrm{CDCl}_{3}\right) \delta(\mathrm{ppm}): 178.2(\mathrm{C}=\mathrm{O}$, leu), 171.5 ( $\mathrm{C}=\mathrm{O}$, phe), 168.7 ( $\mathrm{C}=\mathrm{O}$, ile), 168.0 ( $\mathrm{C}=\mathrm{O}, \operatorname{Ar} \underline{\mathrm{C} O}$ ), 159.4 ( $\mathrm{C}-2, \mathrm{imz})$, 152.2 (C-4, iba), 139.9 (2C, C-2 and C-6, iba), 146.5 (C-5, imz), 137.8 (C-1, iba), 137.2 (C-4, imz), 134.6 (C-y, phe), 130.9 (2C, C-o, phe), 130.2 (2C, C-m, phe), 128.5 (C-p, phe), 105.6 (2C, C-3 and C-5, iba), 58.4 (C- $\alpha$, ile), $52.3\left(\mathrm{O}^{\mathrm{C}} \mathrm{H}_{3}\right), 50.7$ (C- $\alpha$, leu), 49.5 (C- $\alpha$, phe), 45.2 (C- $\beta$, leu), 38.8 (C- $\beta$, phe), 36.5 (C- $\beta$, ile), 31.2 (C$\gamma$, ile), 26.4 (C-y, leu), 25.6 (2C, C- $\delta$, leu), $17.9\left(\beta-\underline{C}_{3}, i m z\right), 14.4\left(C-\gamma^{\prime}\right.$, ile), 10.5 (C- $\delta$, ile); MASS $\mathrm{m} / \mathrm{z}$ (\% rel. int.): 843(2.3), 842(3.8), 841( $\left.\mathrm{M}^{+}, 4.5\right), 826(12.5)$, 810(33.6), 782(10.9), 697(59.2), 669(18.7), 550(100), 522(25.8), 437(65.4), 408(24.5), 328(10.9), 203(11.7), 126(6.5), 120(11.5), 91(12.4), 86(19.5), 82(13.1), 81(10.4), 65(17.2), 59(16.5), 57(14.2), 56(17.2), 55(11.6), 43(16.2), 42(11.7), 31(14.4), 29(10.2), 15(3.7).

\section{Methyl N-[3,5-diiodo-4-(2-methyl-1H-imidazol-5-yl)benzoyl]glycyl-L-histidyl-L- alanyl-L-tryptophanate (2h)}

IR $\left(\mathrm{CHCl}_{3}\right) \vee\left(\mathrm{cm}^{-1}\right): 3489-3483(\mathrm{~N}-\mathrm{H}$ str, rings), 3078-3073, 3053 ( $\mathrm{Ar}-\mathrm{H} \mathrm{str})$, 2964, 2872, 2869 (C-H str, $\mathrm{CH}_{3}$ ), 2929-2922, 2855, 2850 (C-H str, $\left.\mathrm{CH}_{2}\right), 1754$ ( $\mathrm{C}=\mathrm{O}$ str, ester), 1645-1641 ( $\mathrm{C}=\mathrm{O}$ str, amide), 1589-1584, 1428-1423 (skeletal bands), 1538-1533 ( $\mathrm{N}-\mathrm{H}$ def, amide), 1272 (C-O str, ester), 869-864, 838-835, 749 (C-H def, oop), 590 (C-I str); ${ }^{1} \mathrm{H}-\mathrm{NMR}$ (DMSO-d $\left.{ }_{6}\right) \delta$ (ppm): $9.10(1 \mathrm{H}, \mathrm{br} . \mathrm{s}, \mathrm{N} \underline{\mathrm{H}}$, gly), $8.58(3 \mathrm{H}$, br. s, $\mathrm{N} \underline{\mathrm{H}}, \mathrm{imz}$, his and try), $8.42(2 \mathrm{H}, \mathrm{s}, \mathrm{H}-2$ and $\mathrm{H}-6, \mathrm{iba}), 8.30(1 \mathrm{H}$,

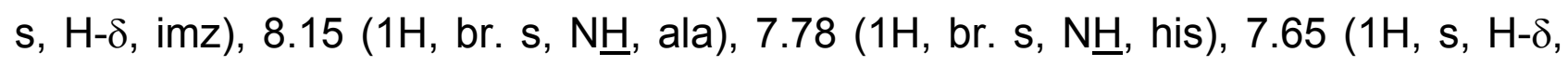


imz, his), $7.52(1 \mathrm{H}, \mathrm{d}, \mathrm{J}=7.75 \mathrm{~Hz}, \mathrm{H}-2$, try), $7.36(1 \mathrm{H}, \mathrm{d}, \mathrm{J}=7.8 \mathrm{~Hz}, \mathrm{H}-2$, his $), 7.15-$

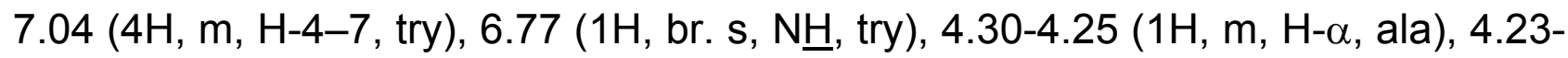
$4.17(1 \mathrm{H}, \mathrm{m}, \mathrm{H}-\alpha$, try $), 4.15-4.11(1 \mathrm{H}, \mathrm{m}, \mathrm{H}-\alpha$, his $), 4.08(2 \mathrm{H}, \mathrm{d}, J=5.1 \mathrm{~Hz}, \mathrm{H}-\alpha$, gly), $3.57\left(3 \mathrm{H}, \mathrm{s}, \mathrm{OC}_{\underline{3}}\right), 3.05-2.96(4 \mathrm{H}, \mathrm{m}, \mathrm{H}-\beta$, his and try $), 2.66\left(3 \mathrm{H}, \mathrm{s}, \beta-\underline{C}_{3}\right.$, imz), $1.49\left(3 \mathrm{H}, \mathrm{d}, \mathrm{J}=5.85 \mathrm{~Hz}, \mathrm{H}-\beta\right.$, ala); ${ }^{13} \mathrm{C}-\mathrm{NMR}\left(\mathrm{CDCl}_{3}\right) \delta$ (ppm): 177.8 (C=O, gly), 175.2 ( $\mathrm{C}=\mathrm{O}, \operatorname{try}), 174.4$ ( $\mathrm{C}=\mathrm{O}$, ala), 170.7 ( $\mathrm{C}=\mathrm{O}, \operatorname{Ar} \underline{\mathrm{CO}}), 169.9$ ( $\mathrm{C}=\mathrm{O}$, his), 159.1 (C-2, imz), 152.0 (C-4, iba), 148.6 (C-2, his), 146.2 (C-5, imz), 141.4 (2C, C-2 and C-6, iba), 137.7 (C-2', try), 136.8 (C-4, imz), 136.0 (C-1, iba), 135.6 (C-4, his), 129.3 (C-3', try), 122.8 (C-2, try), 121.4, 119.7, 118.2 (3C, C-4-6, try), 117.1 (C-5, his), 112.4, 109.5 (2C, C-7 and C-3, try), 105.2 (2C, C-3 and C-5, iba), 63.2 (C- $\alpha$, his), 60.5 ( $\mathrm{C}-\alpha$, ala), 55.6 (C- $\alpha$, try), $51.7\left(\mathrm{O}_{\mathrm{C}} \mathrm{H}_{3}\right), 48.2$ (C- $\alpha$, gly), 28.5 (C- $\beta$, try), 20.1 (C- $\beta$, ala), 19.6 (C- $\beta$, his), 18.7 ( $\left.\beta-\underline{C}_{3}, \mathrm{imz}\right)$; MASS $\mathrm{m} / \mathrm{z}$ (\% rel. int.): 920(2.5), 919( $\left.\mathrm{M}^{+}, 3.6\right)$, 904(14.9), 888(30.9), 860(10.7), 702(59.8), 674(16.6), 631(100), 603(20.4), 494(72.5), 466(23.3), 437(38.6), 408(12.7), 328(10.6), 203(12.2), 159(17.4), 130(9.9), 126(8.8), 116(8.5), 110(14.8), 82(13.3), 81(19.7), 67(5.9), 59(15.8), 56(17.4), 55(9.6), 44(12.2), 31(10.9), 30(5.7), 15(3.4).

\section{Preparation of 3,5-diiodo-4-(2-methyl-1H-imidazol-5-yl)benzoyl amino acids and peptides (3a-d)}

To a solution of the amino acid/peptide methyl esters $(0.01 \mathrm{~mol})$ in THF : $\mathrm{H}_{2} \mathrm{O}$ (1 : $1,36 \mathrm{ml})$, $\mathrm{LiOH}(0.36 \mathrm{~g}, 0.015 \mathrm{~mol})$ was added at $0{ }^{\circ} \mathrm{C}$. The mixture was stirred at $\mathrm{RT}$ for $1 \mathrm{~h}$ and then acidified to $\mathrm{pH} 3.5$ with $1 \mathrm{~N} \mathrm{H}_{2} \mathrm{SO}_{4}$. The aqueous layer was extracted with diethyl ether $(3 \times 25 \mathrm{ml})$. The combined organic extracts were dried over anhydrous $\mathrm{Na}_{2} \mathrm{SO}_{4}$ and concentrated under reduced pressure. The crude products were crystallized from methanol and ether to get hydrolyzed peptide derivatives.

\section{3,5-Diiodo-4-(2-methyl-1H-imidazol-5-yl)benzoyl-L-leucine (3a)}

$\mathrm{IR}(\mathrm{KBr}) v\left(\mathrm{~cm}^{-1}\right): 3486(\mathrm{~N}-\mathrm{H}$ str, ring $), 3298-2508(\mathrm{O}-\mathrm{H}$ str, $\mathrm{COOH}), 3137(\mathrm{NH}$ str, amide), 3078, 3046 (Ar-H str), 2965 (C-H str, $\mathrm{CH}_{3}$ ), 2927, 2852 (C-H str, $\mathrm{CH}_{2}$ ), 
$1713(\mathrm{C}=\mathrm{O}$ str, $\mathrm{COOH}), 1644$ ( $\mathrm{C}=\mathrm{O}$ str, amide), 1588, 1423 (skeletal bands), 1536 ( $\mathrm{N}-\mathrm{H}$ def, amide), 1406 (O-H def, $\mathrm{COOH}), 1387,1360$ (C-H bend, propyl- $i$ ), 860, 836, 759 (C-H def, oop), 585 (C-I str); ${ }^{1} \mathrm{H}-\mathrm{NMR}$ (DMSO-d $\left.{ }_{6}\right) \delta$ (ppm): 10.05 (2H, br. $\mathrm{s}, \mathrm{N} \underline{\mathrm{H}}, \mathrm{imz}$ and $\mathrm{O} \underline{\mathrm{H}}, \mathrm{COO} \underline{\mathrm{H}}), 8.32(2 \mathrm{H}, \mathrm{s}, \mathrm{H}-2$ and $\mathrm{H}-6, \mathrm{iba}), 8.30(1 \mathrm{H}, \mathrm{s}, \mathrm{H}-\delta, \mathrm{imz})$, $8.07(1 \mathrm{H}$, br. s, $\mathrm{N} \underline{\mathrm{H}}, \mathrm{leu}), 4.38-4.33(1 \mathrm{H}, \mathrm{m}, \mathrm{H}-\alpha, \mathrm{leu}), 2.69\left(3 \mathrm{H}, \mathrm{s}, \beta-\underline{\mathrm{H}}_{3}, \mathrm{imz}\right)$, 1.98-1.85 (1H, m, H- $\gamma$, leu), $1.55(2 \mathrm{H}, \mathrm{t}, \mathrm{H}-\beta$, leu $), 0.97(6 \mathrm{H}, \mathrm{d}, \mathrm{J}=6.15 \mathrm{~Hz}, \mathrm{H}-\delta$, leu); ${ }^{13} \mathrm{C}-N M R\left(\mathrm{CDCl}_{3}\right) \delta$ (ppm): 175.0 (C=O, leu), 168.2 (C=O, ArçO), 159.0 (C-2, imz), 154.5 (C-4, iba), 146.4 (C-5, imz), 140.2 (2C, C-2 and C-6, iba), 137.6 (C-4, imz), 136.6 (C-1, iba), 106.7 (2C, C-3 and C-5, iba), 49.2 (C- $\alpha$, leu), 39.9 (C- $\beta$, leu), 24.4 (C- $\gamma$, leu), 19.9 (2C, C- $\delta$, leu), $18.3\left(\beta-\underline{C H}_{3}, \mathrm{imz}\right)$.

\section{3,5-Diiodo-4-(2-methyl-1H-imidazol-5-yl)benzoyl-L-histidyl-L-tyrosine (3b)}

IR $(\mathrm{KBr}) \vee\left(\mathrm{cm}^{-1}\right)$ : 3489, $3482(\mathrm{~N}-\mathrm{H}$ str, rings), $3356(\mathrm{O}-\mathrm{H}$ str, $\mathrm{Ar}-\mathrm{OH}), 3294-$ 2503 (O-H str, $\mathrm{COOH}$ ), 3077-3072, 3054 (Ar-H str), 2965, 2870 (C-H str, $\mathrm{CH}_{3}$ ), 2930, 2929, 2852, 2848 (C-H str, $\mathrm{CH}_{2}$ ), 1708 (C=O str, $\left.\mathrm{COOH}\right), 1644-1640$ (C=O str, amide), 1590-1587, 1425-1419 (skeletal bands), 1409 (O-H def, COOH), 866862, 839, 789, 754, 651 (C-H def, oop), 585 (C-I str); ${ }^{1} \mathrm{H}-\mathrm{NMR}\left(\mathrm{CDCl}_{3}\right) \delta$ (ppm): $9.38(1 \mathrm{H}$, br. $\mathrm{s}, \mathrm{N} \underline{\mathrm{H}}, \mathrm{his}), 8.69(4 \mathrm{H}$, br. $\mathrm{s}, \mathrm{N} \underline{\mathrm{H}}, \mathrm{imz}$, his and $\mathrm{O} \underline{\mathrm{H}}, \mathrm{tyr}, \mathrm{COO} \underline{\mathrm{H}}), 8.42$ $(2 \mathrm{H}, \mathrm{s}, \mathrm{H}-2$ and $\mathrm{H}-6, \mathrm{iba}), 8.32(1 \mathrm{H}, \mathrm{s}, \mathrm{H}-\delta, \mathrm{imz}), 7.65(1 \mathrm{H}, \mathrm{s}, \mathrm{H}-4, \mathrm{his}), 7.39(1 \mathrm{H}, \mathrm{d}$, $J=7.8 \mathrm{~Hz}, \mathrm{H}-2$, try $), 7.25(1 \mathrm{H}$, br. s, $\mathrm{N} \underline{\mathrm{H}}, \operatorname{tyr}), 7.17(2 \mathrm{H}, \mathrm{dd}, \mathrm{J}=8.65,5.3 \mathrm{~Hz}, \mathrm{H}-\mathrm{o}$, tyr), $6.79(2 \mathrm{H}, \mathrm{dd}, \mathrm{J}=8.7,4.95 \mathrm{~Hz}, \mathrm{H}-\mathrm{m}, \mathrm{tyr}), 5.80-5.75(1 \mathrm{H}, \mathrm{m}, \mathrm{H}-\alpha$, his $), 4.20$ $4.15(1 \mathrm{H}, \mathrm{m}, \mathrm{H}-\alpha, \operatorname{tyr}), 3.10(2 \mathrm{H}, \mathrm{d}, \mathrm{J}=5.5 \mathrm{~Hz}, \mathrm{H}-\beta, \operatorname{tyr}), 2.90(2 \mathrm{H}, \mathrm{d}, \mathrm{J}=5.85 \mathrm{~Hz}, \mathrm{H}-$ $\beta$, try), $2.65\left(3 \mathrm{H}, \mathrm{s}, \beta-\underline{\mathrm{H}}_{3}, \mathrm{imz}\right) ;{ }^{13} \mathrm{C}-\mathrm{NMR}\left(\mathrm{CDCl}_{3}\right) \delta(\mathrm{ppm}): 173.4(\mathrm{C}=\mathrm{O}, \mathrm{tyr}), 171.6$ (C=O, his), 171.2 ( $\mathrm{C}=\mathrm{O}, \operatorname{Ar} \underline{\mathrm{C} O}$ ), 159.2 (C-2, imz), 154.4 (C-p, tyr), 151.6 (C-4, iba), 149.3 (C-2, his), 146.2 (C-5, imz), 142.1 (2C, C-m, tyr), 139.6 (2C, C-2 and C-6, iba), 137.1 (C-4, imz), 136.4 (C-1, iba), 135.0 (C-4, his), 128.8 (2C, C-o, tyr), 127.2 (C- $\gamma$, tyr), 117.0 (C-5, his), 105.2 (2C, C-3 and C-5, iba), 65.5 (C- $\alpha$, his), 54.3 (C- $\alpha$, tyr), 37.7 (C- $\beta$, tyr), 19.8 (C- $\beta$, his), $18.6\left(\beta-\underline{C} \mathrm{H}_{3}, \mathrm{imz}\right)$. 


\section{3,5-Diiodo-4-(2-methyl-1H-imidazol-5-yl)benzoyl-L-isoleucyl-L-phenylalanyl-L-} leucine (3c)

IR $\left(\mathrm{CHCl}_{3}\right) \vee\left(\mathrm{cm}^{-1}\right): 3487(\mathrm{~N}-\mathrm{H}$ str, ring), 3308-2513 (O-H str, $\mathrm{COOH}), 3077$, 3050-3046 (Ar-H str), 2971, 2966, 2872 (C-H str, $\left.\mathrm{CH}_{3}\right)$, 2928-2922, 2856-2853 (C-H str, $\mathrm{CH}_{2}$ ), 1711 (C=O str, $\left.\mathrm{COOH}\right), 1646-1640$ (C=O str, amide), 1588, 1420 (skeletal bands), 1536-1530 ( $\mathrm{N}-\mathrm{H}$ def, amide), 1408 (O-H def, $\mathrm{COOH}), 1388,1362$ (C-H bend, propyl-i), 867, 830, 785-780, 749 (C-H def, oop), 588 (C-I str); ${ }^{1} \mathrm{H}-$ NMR (DMSO- $\left.d_{6}\right) \delta$ (ppm): $10.05(2 \mathrm{H}$, br. s, $\underline{N} \underline{H}, \mathrm{imz}$ and $\underline{\mathrm{O}}, \mathrm{COO} \underline{\mathrm{H}}), 8.41(2 \mathrm{H}, \mathrm{s}$, $\mathrm{H}-2$ and $\mathrm{H}-6$, iba), $8.30(1 \mathrm{H}, \mathrm{s}, \mathrm{H}-\delta, \mathrm{imz}), 7.99(1 \mathrm{H}, \mathrm{br} . \mathrm{s}, \mathrm{N} \underline{\mathrm{H}}, \mathrm{phe}), 7.22(2 \mathrm{H}, \mathrm{tt}, \mathrm{J}=$

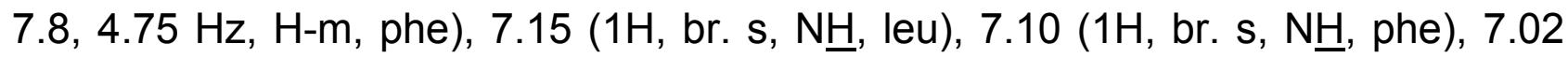
$(1 \mathrm{H}, \mathrm{t}, \mathrm{J}=6.15 \mathrm{~Hz}, \mathrm{H}-\mathrm{p}, \mathrm{phe}), 6.82(2 \mathrm{H}, \mathrm{dd}, \mathrm{J}=8.75,4.15 \mathrm{~Hz}, \mathrm{H}-\mathrm{o}$, phe $), 4.84(1 \mathrm{H}$, q, $\mathrm{H}-\alpha$, phe), $4.58(1 \mathrm{H}, \mathrm{dd}, \mathrm{J}=5.4,3.75 \mathrm{~Hz}, \mathrm{H}-\alpha$, ile), $3.65(1 \mathrm{H}, \mathrm{q}, \mathrm{H}-\alpha$, leu $), 2.82$ $\left(2 \mathrm{H}, \mathrm{d}, J=5.65 \mathrm{~Hz}, \mathrm{H}-\beta\right.$, phe), $2.66\left(3 \mathrm{H}, \mathrm{s}, \beta-\mathrm{C}_{3}, \mathrm{imz}\right), 2.05-1.98(1 \mathrm{H}, \mathrm{m}, \mathrm{H}-\beta$, ile $)$, 1.95-1.88 (1H, m, H-y, leu), 1.67-1.55 (4H, m, H-y, ile and $\mathrm{H}-\beta$, leu $), 1.05(3 \mathrm{H}, \mathrm{d}, \mathrm{J}=$ $5.85 \mathrm{~Hz}, \mathrm{H}-\gamma^{\prime}$, ile), $0.99(3 \mathrm{H}, \mathrm{d}, J=5.95 \mathrm{~Hz}, \mathrm{H}-\delta$, ile), $0.94(6 \mathrm{H}, \mathrm{d}, J=6.3 \mathrm{~Hz}, \mathrm{H}-\delta$, leu); ${ }^{13} \mathrm{C}-N M R\left(\mathrm{CDCl}_{3}\right) \delta$ (ppm): $174.3(\mathrm{C}=\mathrm{O}$, leu), $171.0(\mathrm{C}=\mathrm{O}$, phe), 168.9 ( $\mathrm{C}=\mathrm{O}$, ile), 168.4 (C=O, ArCO), 159.7 (C-2, imz), 152.0 (C-4, iba), 140.4 (2C, C-2 and C-6, iba), 146.0 (C-5, imz), 137.5 (C-1, iba), 136.8 (C-4, imz), 135.2 (C-y, phe), 130.7 (2C, C-o, phe), 130.0 (2C, C-m, phe), 128.2 (C-p, phe), 105.4 (2C, C-3 and C-5, iba), 58.6 (C- $\alpha$, ile), 51.1 (C- $\alpha$, leu), 50.7 (C- $\alpha$, phe), 39.9 (C- $\beta$, leu), 38.6 (C- $\beta$, ile), 38.0 (C- $\beta$, phe), 32.3 (C-y, ile), 24.8 (C-y, leu), 22.1 (2C, C- $\delta$, leu), $18.3\left(\beta-\underline{C}_{3}\right.$,

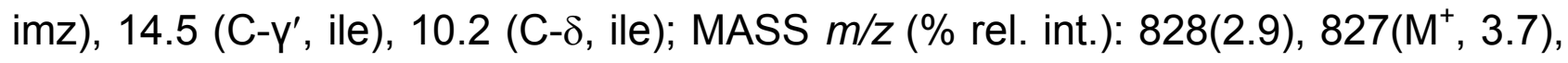
810(30.9), 782(12.5), 697(55.7), 669(19.2), 550(100), 522(23.7), 437(68.5), 408(22.9), 328(11.2), 203(10.9), 126(8.7), 120(10.6), 91(10.9), 86(18.2), 82(13.7), 81(11.2), 65(15.7), 57(13.6), 56(16.4), 55(10.9), 45(13.5), 43(15.5), 42(12.2), 29(9.8), 17(3.8), 15(4.2).

\section{N-[3,5-Diiodo-4-(2-methyl-1H-imidazol-5-yl)benzoyl]glycyl-L-histidyl-L-alanyl- L-tryptophan (3d)}


IR $\left(\mathrm{CHCl}_{3}\right) v\left(\mathrm{~cm}^{-1}\right): 3487-3482(\mathrm{~N}-\mathrm{H}$ str, rings), 3295-2502 (O-H str, $\mathrm{COOH})$, 3077-3072, 3050 (Ar-H str), 2965, 2870, 2869 (C-H str, $\mathrm{CH}_{3}$ ), 2927-2920, 2855, 2852 ( $\mathrm{C}-\mathrm{H}$ str, $\mathrm{CH}_{2}$ ), 1709 ( $\mathrm{C}=\mathrm{O}$ str, $\left.\mathrm{COOH}\right), 1644-1640$ ( $\mathrm{C}=\mathrm{O}$ str, amide), 15891585, 1427-1422 (skeletal bands), 1536-1532 ( $\mathrm{N}-\mathrm{H}$ def, amide), 1405 (O-H def, $\mathrm{COOH}$ ), 867-863, 839-834, 747 (C-H def, oop), 586 (C-I str); ${ }^{1} \mathrm{H}-\mathrm{NMR}$ (DMSO-d $d_{6}$ ) $\delta$ (ppm): 9.45 (4H, br. s, Nㅡ, imz, his, try and $\mathrm{OH}, \mathrm{COO} \underline{\mathrm{H}}), 9.11(1 \mathrm{H}$, br. s, $\mathrm{N} \underline{\mathrm{H}}, \mathrm{gly})$,

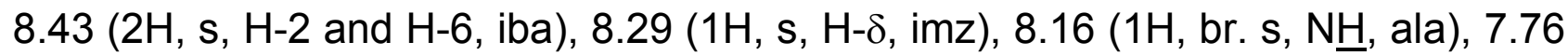

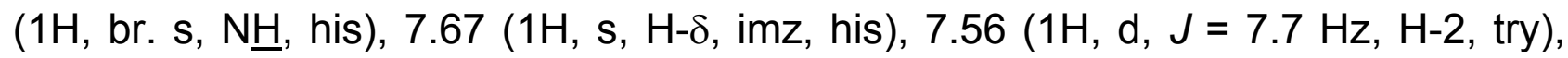
$7.53(1 \mathrm{H}$, br. s, N트, try), $7.38(1 \mathrm{H}, \mathrm{d}, \mathrm{J}=7.75 \mathrm{~Hz}, \mathrm{H}-2$, his), 7.13-6.98 $(4 \mathrm{H}, \mathrm{m}, \mathrm{H}-4-$ 7, try), 4.77-4.72 (1H, m, H- $\alpha$, ala), 4.48-4.42 (1H, m, H- $\alpha$, try), 4.16-4.12 $(1 \mathrm{H}, \mathrm{m}, \mathrm{H}-$ $\alpha$, his), $4.10(2 \mathrm{H}, \mathrm{d}, \mathrm{J}=5.15 \mathrm{~Hz}, \mathrm{H}-\alpha$, gly), 3.08-2.97 (4H, $\mathrm{m}, \mathrm{H}-\beta$, his and try), 2.67 $\left(3 \mathrm{H}, \mathrm{s}, \beta-\mathrm{C}_{3}, \mathrm{imz}\right), 1.51(3 \mathrm{H}, \mathrm{d}, \mathrm{J}=5.9 \mathrm{~Hz}, \mathrm{H}-\beta$, ala $) ;{ }^{13} \mathrm{C}-\mathrm{NMR}\left(\mathrm{CDCl}_{3}\right) \delta(\mathrm{ppm})$ : $178.3(\mathrm{C}=\mathrm{O}$, gly), 172.8 ( $\mathrm{C}=\mathrm{O}$, ala), 172.3 ( $\mathrm{C}=\mathrm{O}$, try), 170.8 ( $\mathrm{C}=\mathrm{O}, \mathrm{Ar} \underline{\mathrm{CO}}), 170.2$ (C=O, his), 159.3 (C-2, imz), 151.7 (C-4, iba), 149.2 (C-2, his), 145.9 (C-5, imz), 141.8 (2C, C-2 and C-6, iba), 138.5 (C-2', try), 137.4 (C-4, imz), 136.7 (C-1, iba), 135.3 (C-4, his), 128.9 (C-3', try), 124.4 (C-2, try), 123.1, 119.4, 118.0 (3C, C-4-6, try), 116.8 (C-5, his), 113.6, 110.5 (2C, C-7 and C-3, try), 105.7 (2C, C-3 and C-5, iba), 60.0 (C- $\alpha$, ala), 59.2 (C- $\alpha$, his), 54.3 (C- $\alpha$, try), 49.1 (C- $\alpha$, gly), 28.8 (C- $\beta$, try), 20.6 (C- $\beta$, ala), 19.8 (C- $\beta$, his), 18.9 ( $\beta-\underline{C}_{3}$, imz); MASS $m / z$ (\% rel. int.): 906(3.3), 905( $\left.\mathrm{M}^{+}, 4.2\right), 888(28.9), 860(11.9), 702(55.9), 674(15.2), 631(100), 603(23.2)$, 494(70.2), 466(23.9), 437(36.8), 408(11.9), 328(11.7), 203(10.5), 159(16.7), 130(8.7), 126(7.9), 116(9.3), 110(13.6), 82(12.8), 81(17.9), 67(6.2), 56(16.8), 55(7.9), 45(11.9), 44(11.6), 30(4.9), 17(3.6), 15(3.9).

\section{Conclusion}

Present investigation describes successful synthesis of title compounds via coupling reaction in good yields. DCC proved to be effective coupling agent both economically and yieldwise, in comparison to DIPC. Greater antifungal activity was found in derivatives with tryptophan and histidine constituents in their amino acid 
chain. Gram negative bacteria were found to be more sensitive in comparison to gram positive bacteria towards the newly synthesized peptide derivatives. Hydrolyzed peptide derivatives exhibited more antimicrobial activity when compared to corresponding methyl ester analogs. Among tested compounds, 2c, $\mathbf{2 d}, \mathbf{2 e}, \mathbf{2 f}, \mathbf{2 h}, \mathbf{3 b}$ and $\mathbf{3 d}$ possessed better antimicrobial activity as compared to standard drugs. On passing toxicity tests, these compounds may prove good candidates for clinical studies and may be potential antifungal and antibacterial agents of future.

\section{Acknowledgement}

Appreciation is expressed to USIC, DU, Delhi (India) and CDRI, Lucknow (India) for spectral and elemental analysis.

\section{References}

[1] De Luca L.

Naturally occurring and synthetic imidazoles: their chemistry and their biological activities.

Curr Med Chem. 2006; 13: 1-23.

[doi:10.2174/092986706775197980]

[2] Congiu C, Cocco M T, Onnis V.

Design, synthesis, and in vitro antitumor activity of new 1,4-diarylimidazole-2ones and their 2-thione analogues.

Bioorg Med Chem Lett. 2008; 18: 989-993.

[doi:10.1016/j.bmcl.2007.12.023]

[3] Li F, Cui J, Guo L, Qian X, Ren W, Wang K, Liu F. Molecular design, chemical synthesis, and biological evaluation of '4-1' pentacyclic aryl/heteroaryl-imidazonaphthalimides.

Bioorg Med Chem. 2007; 15: 5114-5121.

[doi:10.1016/j.bmc.2007.05.032]

[4] Al-Soud Y A, Al-Masoudi N A, Hassan H G, Clercq E D, Pannecouque C. Nitroimidazoles. V. Synthesis and anti-HIV evaluation of new 5-substituted piperazinyl-4-nitroimidazole derivatives.

Acta Pharm. 2007; 57: 379-393.

[doi:10.2478/v10007-007-0031-7] 
[5] Nagarapu L, Satyender A, Rajashaker B, Srinivas K, Rani P R, Radhika K, Subhashini G.

Synthesis and antimicrobial activity of novel C-linked imidazole glycoconjugates.

Bioorg Med Chem Lett. 2008; 18: 1167-1171.

[doi:10.1016/j.bmcl.2007.11.118]

[6] Plachta D A, Baranowski A M, Laudy A E, Starosciak B J, Kleps J.

Synthesis of 1-\{4-[4-(adamant-1-yl)phenoxymethyl]-2-(4-bromophenyl)-1,3dioxolan-2-ylmethyl\}imidazole with expected antifungal and antibacterial activity.

Acta Pol Pharm. 2007; 64: 535-541.

[7] Castellano S, Stefancich G, Chillotti A, Poni G.

Synthesis and antimicrobial properties of 3-aryl-1-(1,1'-biphenyl-4-yl)-2-(1Himidazol-1-yl)propanes as 'carba-analogues' of the $\mathrm{N}$-arylmethyl- $\mathrm{N}-[(1,1$ 'biphenyl)-4-ylmethyl])-1H-imidazol-1-amines, a new class of antifungal agents.

Farmaco. 2003; 58: 563-568.

[doi:10.1016/S0014-827X(03)00094-6]

[8] Perillo I, Repetto E, Caterina M C, Massa R, Gutkind G, Salerno A.

Synthesis, spectroscopic and biological properties of bis(3-arylimidazolidinyl1 )methanes. A novel family of antimicrobial agents.

Eur J Med Chem. 2005; 40: 811-815.

[doi:10.1016/j.ejmech.2005.03.010]

[9] Kelley J L, Thompson J B, Styles V L, Soroko F E, Cooper B R.

Synthesis and anticonvulsant activity of $3 \mathrm{H}$-imidazo[4,5-c]-pyridazine, $1 \mathrm{H}$ imidazo[4,5- $d$ ] pyridazine and $1 \mathrm{H}$-benzimidazole analogues of 9-(2-

fluorobenzyl)-6-methylamino-9H-purine.

J Heterocycl Chem. 1995; 32: 1423-1428.

[10] Gadad A K, Noolvi M N, Karpoormath R V.

Synthesis and anti-tubercular activity of a series of 2-sulfonamido/trifluoro methyl-6-substituted imidazo[2,1-b]-1,3,4-thiadiazole derivatives.

Bioorg Med Chem. 2004; 12: 5651-5659.

[doi:10.1016/j.bmc.2004.07.060]

[11] Benakli K, Terme T, Vanelle P.

Synthesis of new active sulfones in the 5-nitroimidazole series.

Molecules. 2002; 7: 382-385.

[12] Regiec A, Mastalarz H, Miedzybrodzki R, Smietanska K, Jasztold-Howorko R.

Synthesis and biological activity of 4-amino-1-methyl-5-imidazolecarboxylic acid derivatives.

Lett Drug Des Dis. 2006; 3: 192-199.

[doi:10.2174/157018006776286961] 
[13] Wan S B, Chu F M, Guo Z R.

7-imidazolylalkanamido-1-carboxylalkylbenzo-diazepine, a novel series of farnesyltransferase inhibitors.

Yao Xue Xue Bao. 2002; 37: 516-521.

[14] Laufer S, Koch P.

Towards the improvement of the synthesis of novel 4(5)-aryl-5(4)-heteroaryl2-thio-substituted imidazoles and their p38 MAP kinase inhibitory activity.

Org Biomol Chem. 2008; 6: 437-439.

[doi:10.1039/b717605h]

[15] Iradyan M A, Iradyan N S, Stepanyan G M, Arsenyan F G, Paronikyan G M, Darbinyan G A, Kazaryan E V, Garibdzhanyan B T.

Imidazole derivatives. XXIX. Synthesis and biological activity of

thiosemicarbazides and hydrazonohydrazides of 4-nitroimidazole-5-thioacetic acids.

Pharm Chem J. 2003; 37: 67-70.

[doi:10.1023/A:1024077408895]

[16] Gursoy A, lyikosker T, Terzioglu N, Otuk G.

Synthesis and antimicrobial evaluation of some novel

imidazolylmercaptoacetyl thiosemicarbazide and 4-thiazolidinone analogs.

Turk J Pharm Sci. 2005; 2: 1-10.

[17] Dahiya R, Pathak D, Kaur R.

Synthesis, characterization and biological screening of some new aryloxyacetic acid analogs.

$J$ Indian Chem Soc. 2008; 85: in press.

[18] Dahiya R, Kumar A, Yadav R.

Synthesis and biological activity of peptide derivatives of iodoquinazolinones/nitroimidazoles.

Molecules. 2008; 13: 958-976.

[doi:10.3390/molecules13040958]

[19] Dahiya R, Kaur R.

Synthesis of some 1,2,5-trisubstituted benzimidazole analogs as possible anthelmintic and antimicrobial agents.

Int J Biol Chem Sci. 2008; 2: 1-13.

[20] Dahiya R, Pathak D.

Synthetic studies on novel benzimidazolopeptides with antimicrobial, cytotoxic and anthelmintic potential.

Eur J Med Chem. 2007; 42: 772-798.

[doi:10.1016/j.ejmech.2006.11.015] 
[21] Dahiya R, Kaur R.

Synthesis and anthelmintic potential of a novel series of 2-mercaptobenzimidazolopeptides.

Biosci Biotech Res Asia. 2007; 4: 561-566.

[22] Dahiya R, Kaur R.

Synthesis and biological screening of a novel series of 3,4,5-trisubstituted phenoxyacetic acid analogs.

Aust J Basic Appl Sci. 2007; 1: 525-532.

[23] Dahiya R, Pathak D.

Synthesis of novel heterocyclic analogs of 5-(4-methylcarboxamidophenyl)-2furoic acid as potent antimicrobial agents.

Indian J Heterocycl Chem. 2006; 16: 53-56.

[24] Dahiya R, Pathak D, Bhatt S.

Synthesis and biological evaluation of a novel series of 2-(4-chloro-3-methyl phenoxy)acetyl amino acids and peptides.

J Saudi Chem Soc. 2006; 10: 165-176.

[25] Dahiya R, Pathak D.

Synthesis and biological screening of a novel series of 6-nitro-2-(4toluoyl)benzoyl amino acids and peptides.

J Nepal Pharm Assoc. 2006; 14: 17-26.

[26] Dahiya R, Pathak D, Bhatt S.

Synthesis and biological evaluation of a novel series of 2-(2'-isopropyl-5'methylphenoxy)acetyl amino acids and dipeptides.

Bull Chem Soc Ethiop. 2006; 20: 235-245.

[27] Dahiya R, Pathak D.

Synthesis and antimicrobial activity of novel series of 2-aroyl-6-nitrobenzoyl amino acids and peptides.

Orient J Chem. 2006; 22: 123-128.

[28] Himaja M, Rajiv, Ramana M V, Poojary B, Satyanarayana D, Subrahmanyam E V, Bhat KI.

Synthesis and biological activity of a novel series of 4-[2'-(6'-nitro)benzimidazolyl]benzoyl amino acids and peptides.

Boll Chim Farm. 2003; 142: 450-453.

[29] Himaja M, Rajiv, Ramana M V.

Synthesis of 6-nitrobenzimidazol-1-acetyl amino acids and peptides as potent anthelmintic agents.

Indian J Heterocycl Chem. 2002; 12: 121-124.

[30] Vogel A, editor.

Textbook of Practical Organic Chemistry.

Singapore:Pearson Education, 2004: 909-910. 
[31] Bodanzsky M, Bodanzsky A, editors.

The Practice of Peptide Synthesis.

New York:Springer-Verlag, 1984: 143-207.

[32] Bauer A W, Kirby W M, Sherris J C, Turck M.

Antibiotic susceptibility testing by a standardized single disk method. Am J Clin Path. 1966; 45: 493-496. 